\title{
Protrusion of the Infraorbital Nerve into the Maxillary Sinus on CT: Prevalence, Proposed Grading Method, and Suggested Clinical Implications
}

\author{
(D).E. Lantos, A.N. Pearlman, (D)A. Gupta, (D).L. Chazen, R.D. Zimmerman, D.R. Shatzkes, and C.D. Phillips
}

\begin{abstract}
BACKGROUND AND PURPOSE: The infraorbital nerve arises from the maxillary branch of the trigeminal nerve and normally traverses the orbital floor in the infraorbital canal. Sometimes, however, the infraorbital canal protrudes into the maxillary sinus separate from the orbital floor. We systematically studied the prevalence of this variant.
\end{abstract}

MATERIALS AND METHODS: We performed a retrospective review of 500 consecutive sinus CTs performed at our outpatient centers. The infraorbital nerve protruded into the maxillary sinus if the entire wall of the infraorbital canal was separate from the walls of the sinus. We recorded the length of the bony septum that attached the infraorbital canal to the wall of the maxillary sinus and noted whether the protrusion was bilateral. We also measured the distance from the inferior orbital rim where the infraorbital canal begins to protrude into the sinus.

RESULTS: There was a prevalence of $10.8 \%$ for infraorbital canal protrusion into the maxillary sinus and $5.6 \%$ for bilateral protrusion. The median length of the bony septum attaching the infraorbital canal to a maxillary sinus wall, which was invariably present, was $4 \mathrm{~mm}$. The median distance at which the infraorbital nerve began to protrude into the sinus was $11 \mathrm{~mm}$ posterior to the inferior orbital rim.

CONCLUSIONS: Although this condition has been reported in only 3 patients previously, infraorbital canal protrusion into the maxillary sinus was present in $>10 \%$ of our cohort. Identification of this variant on CT could help a surgeon avoid patient injury.

ABBREVIATIONS: $I O C=$ infraorbital canal; $I O N=$ infraorbital nerve

$\mathbf{C}^{\mathrm{T}}$ T of the paranasal sinuses is an important diagnostic technique in the work-up of patients with known or suspected disease of the nasal cavity and paranasal sinuses. CT gives the surgeon a roadmap for surgery and alerts the surgeon to the presence of potentially clinically relevant anatomic variants. Many sinonasal variants are important to identify since their presence may increase the risk of surgical error. ${ }^{1}$ With the advent of endoscopic techniques, surgery of the paranasal sinuses has expanded to involve complex procedures that were once reserved for open approaches. Thus, it is extremely important to identify such variations from the normal sinus anatomy, especially in patients who are likely to require extended

Received March 31, 2015; accepted after revision June 17.

From the Departments of Radiology (J.E.L., A.G., J.L.C., R.D.Z., C.D.P.) and Otolaryngology (A.N.P.), Weill Cornell Medical College, New York, New York; and Department of Radiology (D.R.S.), Lenox Hill Hospital, New York, New York.

Please address correspondence to Joshua E. Lantos, MD, Weill Cornell Medical College, Department of Radiology, 535 E 68th St, New York, NY 10065; e-mail: jol9057@med.cornell.edu

http://dx.doi.org/10.3174/ajnr.A4588 endoscopic sinus surgery for etiologies such as inverted papilloma, mucocele, trauma, or malignant tumor.

The infraorbital nerve is the distal portion of the maxillary nerve (V2), which originates as the second division of the trigeminal nerve (fifth cranial nerve). After the maxillary nerve traverses the foramen rotundum, it enters the pterygopalatine fossa and gives off nasal and palatine branches before exiting through the inferior orbital fissure and terminating as the infraorbital nerve (ION). The ION then enters the infraorbital canal (IOC) through the infraorbital groove. The IOC is a bony canal typically within the orbital process of the maxilla, synonymous with the floor of the orbit. The ION exits the IOC through the infraorbital foramen of the anterior maxilla. Variably, the IOC can protrude into the maxillary sinus separate from the floor of the orbit. This may leave the ION susceptible to injury during endoscopic or open sinus surgery. To date, just 3 case reports exist in the literature describing this variant, ${ }^{2,3}$ with no large studies describing the frequency with which it occurs. The aim of this study was to establish the prevalence of infraorbital nerve protrusion into the maxillary sinus and define its common characteristics. This variation is of clinical importance in sinus surgery, and we suggest an accompa- 

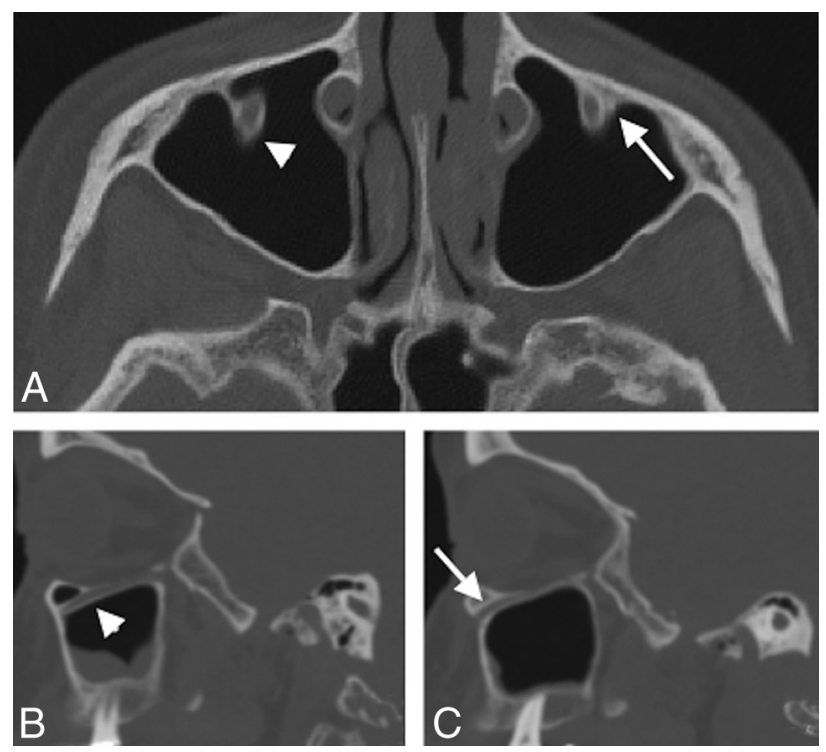

FIG 1. Axial ( $A$ ), right parasagittal ( $B)$, and left parasagittal $(C)$ sinus $C T$ images in a 55-year-old woman show unilateral right-sided protrusion of the ION into the maxillary sinus (arrowhead in $A$ and $B$ ). While part of the wall of the left IOC protrudes into the sinus, the entire circumference of the IOC is not distinct from the anterior maxillary sinus wall; this feature is confirmed on the sagittal image through the left maxillary sinus (arrows in $A$ and $C$ ). Additionally, no measurable bony septum connects the IOC to the wall of the maxillary sinus. This distinction was chosen to define protrusion of the ION into the maxillary sinus.

nying grading scale to relay the degree of protrusion to the surgeon.

\section{MATERIALS AND METHODS}

A retrospective image review of sinus CTs performed at our institution was conducted after obtaining institutional review board approval, including a waiver for informed consent. Five hundred consecutive sinus CTs performed on 500 distinct patients at our outpatient centers from February 2, 2014 to July 31, 2014 were included in the study. Most patients had known or suspected sinus inflammatory disease and were referred by an otorhinolaryngologist. All CTs reviewed were acquired in the axial plane at 0.625 $\mathrm{mm}$ and reconstructed in a bone algorithm at $1.25 \mathrm{~mm}$ in the axial plane. Sagittal and coronal reconstructions in either bone or softtissue algorithms at $1.5-\mathrm{mm}$ thickness were also performed on all studies. Coverage on all CT scans was from the vertex of the skull through the level of the hard palate; expanded coverage was performed to fuse data with endoscopic instrumentation in these potential surgical candidates. No CT scans were excluded due to technical inadequacy or an inability to identify the IOC.

CTs were performed on one of our several scanners including a LightSpeed Pro-16 or HD-750 scanner (GE Healthcare, Milwaukee, Wisconsin) and were reviewed on a PACS on bone window settings (width/level, 4095/600) by a neuroradiology fellow (J.E.L.). The IOC was considered to protrude into the maxillary sinus if the entire wall of the IOC was not in contact with any of the walls of the maxillary sinus on a single image (Fig 1). When this variant was present and a bony septum connected the IOC with a wall of the maxillary sinus, the maximal depicted length of the septum on axial images was measured (Fig $2 A$ ). In patients
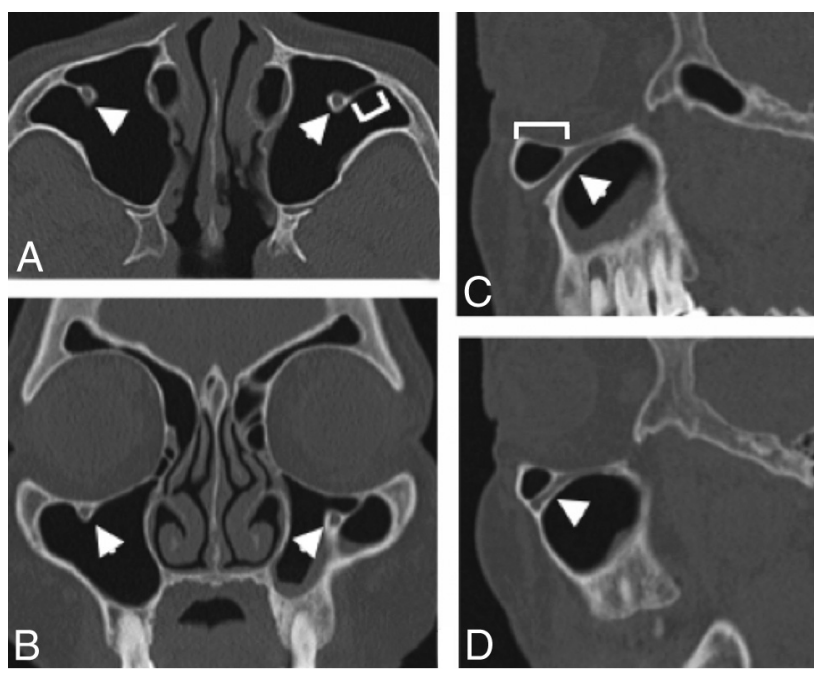

FIG 2. Axial $(A)$, coronal $(B)$, left parasagittal $(C)$, and right parasagittal (D) CT images in a 72-year-old woman show bilateral protrusion of the ION (arrowheads) into the maxillary sinus. The septum attaching the IOC to the anterior wall of the sinus was measured (bracket in A) on the axial image. The distance at which protrusion begins posterior to the inferior orbital rim (bracket in C) was measured on the sagittal image.

\section{The 54 patients composing our group of patients with IOC} protrusion broken down into 3 groups ${ }^{\mathrm{a}}$

\begin{tabular}{lccccc} 
Class & $\begin{array}{c}\text { Septum } \\
\text { Length }\end{array}$ & $\begin{array}{c}\text { No. of } \\
\text { Patients }\end{array}$ & $\begin{array}{c}\text { Median } \\
\text { Age (yr) }\end{array}$ & Female/Male & $\begin{array}{c}\text { Bilateral } \\
(\%)\end{array}$ \\
\hline 1 & $1-3 \mathrm{~mm}$ & 26 & 62.5 & $15: 11$ & $12(46 \%)$ \\
2 & $4-6 \mathrm{~mm}$ & 18 & 48.5 & $9: 9$ & $8(44 \%)$ \\
3 & $7-11 \mathrm{~mm}$ & 10 & 43.5 & $5: 5$ & $8(80 \%)$ \\
\hline
\end{tabular}

a The most medial septum was chosen in patients with multiple septa.

with $>1$ septum, the most medial septum attaching to the anterior wall was measured. The presence of multiple maxillary sinus bony septa attached to the IOC was recorded. We noted from which wall of the maxillary sinus (anterior, posterior, or medial) the septum arose. On sagittal images, we also measured the distance at which the ION began to protrude into the sinus, measuring posteriorly from the fixed anatomic landmark of the inferior orbital rim (Fig $2 C$ ). Note was made of whether the protruding IOC was unilateral or bilateral. Patients with IOC protrusion were divided into 3 classes based on maximal septum length: 1-3; 3-6; and $\geq 7 \mathrm{~mm}$.

\section{RESULTS}

The study population consisted of 272 females (54.4\%) and 228 males $(46.6 \%)$ with a median age of 47 years (range, 6-95 years). Protrusion of the IOC into the maxillary sinus was identified in 54 patients $(10.8 \%)$; it was bilateral in $28(5.6 \%)$ and unilateral in 26 $(5.2 \%)$. All protruding IOCs were anchored to a wall of the maxillary sinus by at least 1 bony septum. The median length of the septum attaching the IOC to the wall of the maxillary sinus was 4 $\mathrm{mm}$ (range, $1-11 \mathrm{~mm}$ ). The median distance posterior to the inferior orbital rim at which the ION began to protrude into the sinus was $11 \mathrm{~mm}$ (range, 5-24 mm). Of the 54 patients with a protruding IOC, $26(48 \%)$ had a bony septum measuring $1-3$ $\mathrm{mm}, 18(33 \%)$ had a bony septum measuring 4-6 $\mathrm{mm}$, and 10 (19\%) had a bony septum measuring $\geq 7 \mathrm{~mm}$ (Table). One patient had a single bony septum attached to the posterior wall of the 
sinus; otherwise, all patients with posterior septa had additional bony attachments to the anterior wall. No patient had a bony attachment to the medial wall of the sinus. A second septum attached to a protruding IOC was present in 9 patients, and a second septum was bilateral in 4 patients. A third septum was present in 1 patient unilaterally.

\section{DISCUSSION}

In our study of 500 consecutive sinus CTs performed at our outpatient centers, we found that IOC protrusion into the maxillary sinus had a prevalence of $10.8 \%$. At present, only 3 case reports exist describing this anatomic variant. ${ }^{2,3}$ Mailleux et $\mathrm{al}^{2}$ described 2 patients: One demonstrated IOC protrusion bilaterally, and 1 had unilateral protrusion. Elnil et $\mathrm{al}^{3}$ described a third patient in whom the finding was bilateral. No formal definition exists for IOC protrusion into the maxillary sinus. Because we aimed to define a clinically relevant variant that may place a patient at greater surgical risk, we considered IOC protrusion into the sinus present if the entire $360^{\circ}$ of the wall of the IOC was not in contact with a wall of the maxillary sinus on at least 1 axial CT image. With this definition, 1 of the 2 patients reported by Mailleux et al may not have had IOC protrusion based on the images provided in that publication.

A bony septum attaching the protruding IOC to a wall of the maxillary sinus was invariably present in our study, and we found a wide range in the length of this septum. The length of this septum is important because the further into the sinus the IOC protrudes, the more susceptible it may be to injury during sinus surgery. When multiple septa were present, we measured the most medial septum attaching to the anterior wall because we thought it gave a more representative measurement of how far into the sinus the IOC protruded. Sometimes lateral attachments were very long and did not reflect the degree of protrusion relative to protruding IOCs with single attachment. Additionally, the medial septum was thought to be more clinically relevant because endoscopic sinus surgery more frequently takes place in the medial maxillary sinus.

At the lower end of our septum length range (1-3 mm), IOC protrusion may not be of clinical significance, and reporting the small distance of protrusion can convey that to the clinician. Of our 500 patients, however, $28(5.6 \%)$ had septa measuring $>3$ $\mathrm{mm}$ and $10(2 \%)$ had septa measuring $>6 \mathrm{~mm}$. It has not yet been established at what length the septum becomes clinically relevant. We chose to group our patients into 3 classes based on the length of the septum attached to the protruding IOC (Table). Possibly only 1 group (class 3 , those with the longest septa), will prove to be clinically relevant, similar to the Keros classification of the length of the lateral lamella of the cribriform plate, in which only type 3 is considered clinically relevant, placing the patient at the highest surgical risk for iatrogenic injury. ${ }^{4}$

We also measured the distance at which the IOC begins to protrude into the maxillary sinus posterior to the inferior orbital rim because the inferior orbital rim is a fixed anatomic landmark and it may be a useful way for a surgeon to conceptualize the degree of protrusion before an intervention. The IOC began to protrude into the sinus more proximally with a longer measurement. As with the length of the bony septum, it is not clear at this

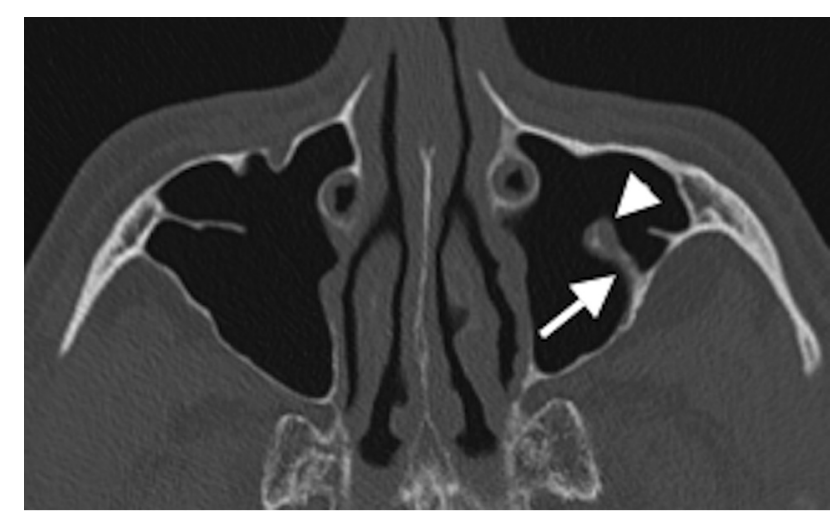

FIG 3. Axial $C T$ image in a 56-year-old man shows left-sided protrusion of the ION into the maxillary sinus (arrowhead) attached to a single posterior septum (arrow). This was the only patient with a septum attaching a protruding IOC to the posterior wall of the maxillary sinus without an additional septum attaching to the anterior wall.

time at what point this measurement becomes clinically relevant. Nevertheless, stating this measurement may give a surgeon an idea of the severity of the protrusion and in what location to expect the nerve when entering the sinus.

The wall to which a septum attaches is important to note as well; while we did not find a patient with an attachment to the medial wall, such a septum could theoretically be at greater risk during maxillary antrostomy. Only 1 patient had a single septum attaching a protruding IOC to the posterior wall (Fig 3), with the remaining patients all having at least 1 such septum attached to the anterior wall.

The significance of a protruding IOC into the maxillary sinus has not been fully addressed in the literature. For a sinonasal variant to be considered relevant, 1 of the following 4 must be true: The variant impairs normal drainage pathways, hinders endoscopic access to distal areas, serves as a focus for occult disease, or increases the risk of surgical error. ${ }^{1}$ Examples of anatomic variants detectable on CT that may increase the risk of iatrogenic injury include sphenoethmoid (Onodi) cells, optic nerve or internal carotid artery protrusion into the sphenoid sinus, insertion of the intersphenoid sinus septum onto the carotid canal, and aeration of the anterior clinoid process. A sphenoethmoid cell is a posterior ethmoid cell that pneumatizes superiorly above the sphenoid sinus and posteriorly beyond the anterior sphenoid face, with the optic nerve being intimately related to its lateral wall. It places the optic nerve at increased risk of injury during posterior ethmoidectomy; the reported incidence of this variant ranges from $8 \%$ to $14 \% .{ }^{5}$ Optic nerve or internal carotid artery canal dehiscence and protrusion into the sphenoid sinus increase the risk of injury to those structures as well. Most of the literature supports a prevalence of optic nerve dehiscence of $0.7 \%-8 \%{ }^{6}$ Reported prevalence for optic nerve protrusion ranges from $8 \%$ to $35 \%$, but no consensus definition exists for what constitutes protrusion, despite effort at developing a classification scheme. ${ }^{7}$ The prevalence of internal carotid artery dehiscence and protrusion varies widely from $1.5 \%$ to $30 \%$ and $5.2 \%$ to $67 \%$, respectively, with variation potentially depending on the study population. ${ }^{6}$ Aeration of the anterior clinoid process has a reported prevalence of $12 \%-19 \%,{ }^{8,9}$ and when optic nerve protrusion is 

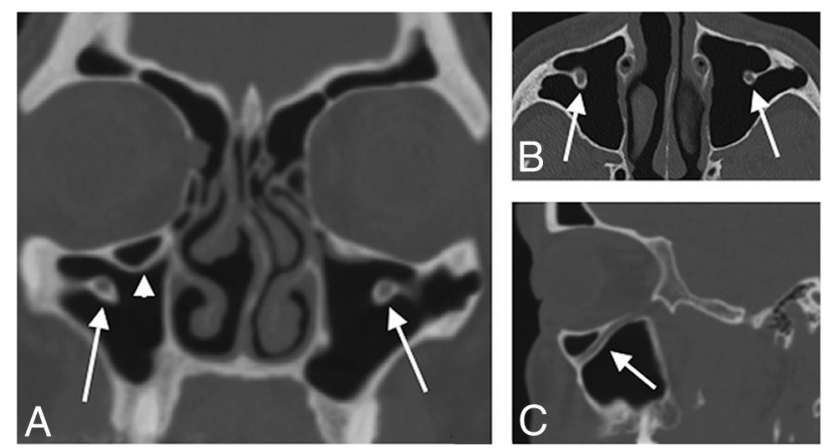

FIG 4. Coronal $(A)$, axial $(B)$, and right parasagittal $(C) C T$ images in a 58-year-old man show bilateral protrusion of the IOC into the maxillary sinus. On the coronal image, an infraorbital ethmoid (Haller) cell is seen on the right (arrowhead) just medial and superior to the protruding IOC (arrow). Bilateral protrusion is seen on the axial image (arrows), and confirmation of protrusion is seen on the sagittal image through the right maxillary sinus. The presence of a protruding IOC near an infraorbital cell is important for the endoscopic surgeon to know preoperatively, in the event that the infraorbital cell is targeted during sinus surgery.

present, there is a $56 \%$ chance of ipsilateral anterior clinoid aeration. ${ }^{9}$ Insertion of an intersphenoid sinus septum onto the carotid canal has been reported in $4.7 \% .{ }^{10}$ The $10.6 \%$ prevalence for the presence of IOC protrusion into the maxillary sinus observed in this study is comparable with these other variants that are commonly accepted as reportable on sinus CT.

Authors of prior case reports of ION protrusion believed that this variant leads to an increased risk during endoscopic sinus surgery; thus, those authors advocated mentioning this variant in preoperative sinus CT reports. ${ }^{2,3}$ The long-term consequence of injury to the ION is best described in the setting of trauma, specifically in zygomaticomaxillary complex fractures, in which injury to the ION occurred in $64.4 \%$ of cases in 1 series of 478 patients with unilateral fractures. ${ }^{11}$ Traumatic ION injury results in ipsilateral paresthesias and numbness of the nose and lip, and we would expect similar outcomes in the setting of iatrogenic injury to a protruding ION during sinus surgery. Iatrogenic ION injury with subsequent paresthesia has been described in rhinoplasty, presumably after the ION exited the maxilla through the infraorbital foramen. ${ }^{12}$ Iatrogenic injury to the ION has also been reported in the setting of endoscopic sinus surgery, requiring canine fossa puncture for insertion of an endoscope or microdebrider for extensive maxillary sinus disease, as seen in polyposis and extensive fungal debris. One review of 37 such canine punctures found evidence of long-term injury to 6 of 37 (16\%) IONs, ${ }^{13}$ including facial pain and paresthesias. While the ION is usually visualized after buccal mucosa incision and lifting of the periosteum, noting ION protrusion on preoperative CT will alert the surgeon to the possibility that the ION could be in an unexpected location.

We suggest that the presence of a protruding IOC should be characterized when there are nearby anatomic variants such as an infraorbital ethmoid (Haller) cell, which are likely to be part of the surgical dissection (Fig 4). As can be seen in Fig 4, this patient has a class 3 protrusion of the IOC and the ION is in close approximation to the infraorbital ethmoid cell. In attempting to adequately resect this cell with curved instrumentation, whether
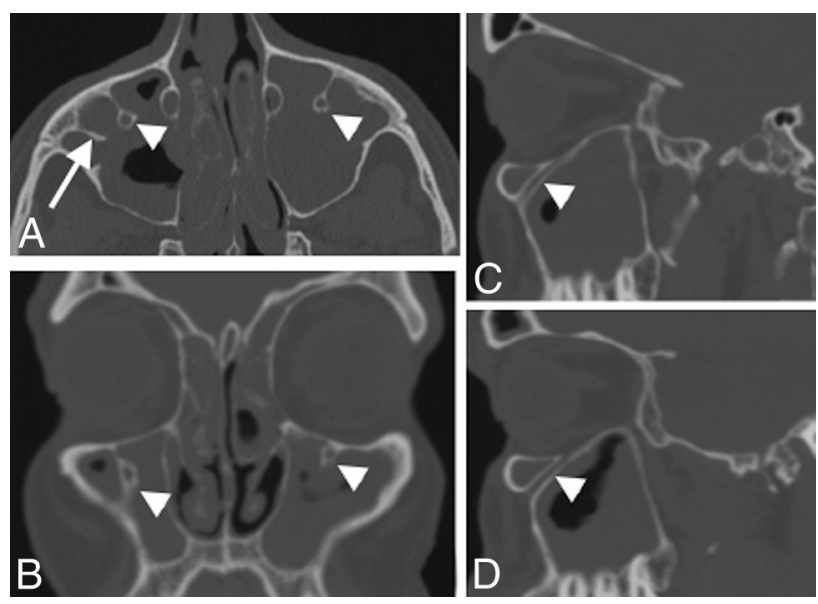

FIG 5. Axial $(A)$, coronal $(B)$, right $(C)$, and left $(D)$ parasagittal $C T$ images of a 42-year-old man show extensive paranasal sinus inflammatory disease and also demonstrate bilateral protrusion of the IOC (arrowheads) into the maxillary sinus. Incidental note is made of an additional bony septum in the right maxillary sinus (arrow in A). For this surgical candidate, the IOC position should be mentioned in the report.

powered or blunt, one could easily inadvertently disrupt the ION in this position. Simply unroofing the bone from the nerve and creating a dehiscence may cause the patient to have neuropathy of the ION. Chandra and Kennedy ${ }^{14}$ described a similar variant to the protruding IOC, in which the IOC coursed within the inferior lamella of an infraorbital ethmoid (Haller) cell rather than within the inferior orbital wall. This location would put the ION at a risk similar to that of a protruding IOC because the IOC is not in the expected location of the orbital floor; thus, the ION is not as well-protected. We did not, however, find this variant in our imaging review of 500 patients.

Other disease processes of the maxillary sinus, such as chronic inflammation or neoplasm, may put the protruding ION at risk. Extensive inflammatory disease within the maxillary sinus may cause the bony canal to be weakened or dehiscent (Fig 5). Antrochoanal polyps can originate from the anterior wall of the maxillary sinus, in the location of many of our protruding IONs and their bony septa, and endoscopic dissection along the anterior aspect of the maxillary sinus requires curved powered tissue debriders and angled telescopes for visualization. Resection of an inverted papilloma requires a wide local excision around its lateral maxillary sinus wall origin due to the $6 \%-10 \%$ rate of malignant transformation. ${ }^{15}$ An endoscopic approach requires the use of angled burrs to remove the bone intimately associated with the origin of the inverted papilloma. In each of these instances, a protruding ION would be at an increased risk of injury.

Open surgical approaches to the maxillary sinus also place the ION at risk of injury. The Caldwell-Luc procedure is an open approach through the gingivobuccal sulcus into the maxillary sinus, most commonly used to address difficult pathology not amenable to endoscopic techniques, such as resection of neoplasm, recurrent antrochoanal polyp, dentigerous cyst, and fungal disease. Access to the orbit and pterygoid space can also be achieved through this approach. ${ }^{16,17}$ Furthermore, an antral punch to introduce a balloon dilation catheter to approach the maxillary infundibulum is being used with increasing frequency. ${ }^{18}$ These 
open, anterior approaches to the maxillary sinus place a protruding ION at increased risk of iatrogenic injury because most are connected to a bony septum attached to the anterior maxillary sinus wall. Thus, we believe that in the presurgical setting, identification of the location of the ION should be part of the routine search pattern for interpreting physicians to reduce the risk of ION injury.

The prevalence of $10.8 \%$ of IOC protrusion in this cohort suggests that it is a common anatomic variation, though its prevalence in the general population not referred for imaging is unknown. Although we have no reason to suspect it, IOC protrusion into the maxillary sinus may be more common in individuals with known or suspected sinus inflammatory disease. In this instance, our prevalence would not be applicable to the general population, and future studies may help confirm that our number is generalizable to the greater population. Furthermore, we suggest a classification that can be used to easily convey the degree of protrusion to the surgeon. At this time, because the rate of iatrogenic injury to the protruding ION is unknown, the clinical utility of this classification scale is not clear. Still, because there are an estimated 250,000 endoscopic sinus surgical procedures per year performed in the United States and the maxillary sinus is the most commonly instrumented sinus, a rate of $10.8 \%$ ION protrusion is of particular clinical relevance. ${ }^{19}$

\section{CONCLUSIONS}

To date, only 3 case reports in the literature have described protrusion of the IOC into the maxillary sinus rather than within the orbital process of the maxilla (orbital floor). Using the definition that the entire wall of the IOC had to be separate from all walls of the maxillary sinus on a single image, we found a prevalence of $10.8 \%$ for this variant during review of $500 \mathrm{CT}$ scans. The distance of protrusion into the sinus should be noted because this information may help avoid iatrogenic injury during surgical intervention.

\section{REFERENCES}

1. Earwalker J. Anatomic variants in sinonasal CT. Radiographics 1993; 13:381-415 CrossRef Medline

2. Mailleux P, Desgain O, Ingabire MI. Ectopic infraorbital nerve in a maxillary sinus septum: another potentially dangerous variant for sinus surgery. JBR-BTR 2010;93:308-09 Medline

3. Elnil H, Al-Tubaikh JA, El Beltagi AH. Into the septum I go, a case of bilateral ectopic infraorbital nerves: a not-to-miss preoperative sinonasal CT variant. Neuroradiol J2014;27:146 - 49 CrossRef Medline

4. Stammberger HR, Kennedy DW; Anatomic Terminology Group. Paranasal sinuses: anatomic terminology and nomenclature. Ann Otol Rhinol Laryngol Suppl 1995;167:7-16

5. Kantarci M, Karasen RM, Alper F, et al. Remarkable anatomic variations in paranasal sinus region and their clinical importance. Eur J Radiol 2004;50:296-302 CrossRef Medline

6. Anusha B, Baharudin A, Phillip R, et al. Anatomical variations of the sphenoid sinus and its adjacent structures: a review of existing literature. Surg Radiol Anat 2014;36:419-27 CrossRef Medline

7. DeLano MC, Fun FY, Zinreich SI. Relationship of the optic nerve to the posterior paranasal sinuses: a CT anatomic study. AJNR Am J Neuroradiol 1996;17:669-75 Medline

8. Avci E, Bandemci G, Ozturk A. Microsurgical landmarks for safe removal of the anterior clinoid process. Minim Invasive Neurosurg 2005;48:268-72 CrossRef Medline

9. Chen YL, Lee LA, Lim KE. Surgical consideration to optic nerve protrusion according to sinus computed tomography. Otolaryngol Head Neck Surg 2006;134:499-505 CrossRef Medline

10. Hamid O, El Fiky L, Hassan O, et al. Anatomic variations of the sphenoid sinus and their impact on trans-sphenoid pituitary surgery. Skull Base 2008;18:9-15 CrossRef Medline

11. Sakavicious D, Juodzybalys G, Kubilius R, et al. Investigation of infraorbital nerve injury following zygomaticomaxillary complex fractures. J Oral Rehabil 2008;35:903-16 CrossRef Medline

12. Meyer M, Moss AL, Cullen KW. Infraorbital nerve palsy after rhinoplasty. J Craniomaxillofac Surg 1990;18:173-74 CrossRef Medline

13. Robinson SR, Baird R, Le T, et al. The incidence of complications after canine fossa puncture performed during endoscopic sinus surgery. Am J Rhinol 2005;19:203-06 Medline

14. Chandra RK, Kennedy DW. Surgical implications of an unusual anomaly of the infraorbital nerve. Ear Nose Throat J 2004;83:766-67 Medline

15. von Buchwald C, Bradley PJ. Risks of malignancy in inverted papilloma of the nose and paranasal sinuses. Curr Opin Otolaryngol Head Neck Surg 2007;15:95-98 CrossRef Medline

16. Barzilai G, Greenberg E, Uri N. Indications for Caldwell-Luc approach in the endoscopic era. Otolaryngol Head Neck Surg 2005;132: 219-20 CrossRef Medline

17. Matheny KE, Duncavage JA. Contemporary indications for the Caldwell-Luc procedure. Curr Opin Otolaryngol Head Neck Surg 2003;11:23-26 CrossRef Medline

18. Stankiewicz J, Tami T, Truitt T, et al. Transantral, endoscopically guided balloon dilatation of the ostiomeatal complex for chronic rhinosinusitis under local anesthesia. Am J Rhinol Allergy 2009;23: 321-27 CrossRef Medline

19. Bhattacharyya N. Ambulatory sinus and nasal surgery in the United States: demographics and perioperative outcomes. Laryngoscope 2010;120:635-38 CrossRef Medline 